Seguridad Operacional

y Logística Aeronáutica

s.

Segurança Operacional

e Logística Aeronáutica

Operational Safety

and Aviation Logistics
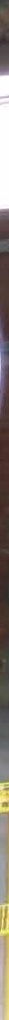

는 $11=-2=$

\title{
TÉCNICA DE PARTÍCULAS MAGNÉTICAS: CASO DEL LABORATORIO DEL CAMAN
}

TÉCNICA DE PARTICULAS MAGNÉTICAS: CASO DO LABORATÓRIO DE CAMAN

Magnetic Particle Technique: Lab Case of CAMAN

Sonia Ruth Rincón Urbina, Daniel Andrés Calvo Cobos, Erika Juliana Estrada Villa

Escuela de Postgrados de la Fuerza Aérea Colombiana

\section{CIENCIA Y PODER AÉREO}

ISSN 1909-7050 / E- ISSN 2389-2468 / Volumen 10 / Enero-Diciembre de 2015/ Colombia/ Pp. 1-260

Recibido: 3/06/2015

Aprobado evaluador interno: 12/10/2015

Aprobado evaluador externo: 20/11/2015

Doi: http://dx.doi.org/10.18667/cienciaypoderaereo.435 
Para citar este artículo:

Rincón, S., Calvo, D., y Estrada, E. (2015). Técnica de partículas magnéticas: caso del laboratorio del CAMAN. Ciencia y Poder Aéreo, 10 (1), 59-70. Doi: http://dx.doi. org/10.18667/cienciaypoderaereo.435

' Reporte de caso, derivado del trabajo de investigación titulado: Manual de Procesos de Inspección de la técnica partículas magnéticas del laboratorio del Comando Aéreo de Mantenimiento - CAMAN, desarrollado por: CT. Sonia Ruth Rincón Urbina y CT. Daniel Andrés Calvo Cobos, en la Especialización en Logística Aeronáutica de la Escuela de Postgrados de la Fuerza Aérea Colombiana.

${ }^{2}$ Case report, derived from the research work entitled: Manual of Process inspection of the magnetic particles technique from CANAM lab - developed by: CT. Sonia Ruth Rincón Urbina and CT. Daniel Calvo Andres Cobos at the specialized studies on Aviation Logistics from the Colombian Air Force's Postgraduate School.

${ }^{3}$ Relato de caso derivado do trabalho de investigação: Manual de Processos de Inspeção da técnica de partículas magnéticas do laboratório Caman - CAMAN desenvolvido por: CT. Sonia Ruth Rincón Urbina e CT. Daniel Calvo Andres Cobos na Especialização da Escola de PósGraduação da Força Aérea Colombiana.

${ }^{4}$ Oficial en el grado de Capitán de la Fuerza Aérea Colombiana. Especialista en Logística Aeronáutica, Maestría Aerospace Manufacturing en curso en Cranfield University, Reino Unido. Correo electrónico: sonitorincon@hotmail.com

${ }^{5}$ Oficial en el grado de Capitán de la Fuerza Aérea Colombiana. Especialista en Logística Aeronáutica. Máster de Ensayos en Vuelo y Certificación de Aeronaves en curso en la Universidad Politécnica de Madrid España. Correo electrónico: andrescalvo13@gmail.com

${ }^{6}$ Docente, investigadora y asesora de trabajos de grado de investigación, Departamento de Investigación de la Escuela de Postgrados de la Fuerza Aérea Colombiana. Magíster en Informática Educativa, Universidad de la Sabana. Correo electrónico: erikajulianaestrada@hotmail.com
Resumen: el presente artículo presenta el procedimiento general para el empleo de la técnica de partículas magnéticas; describe de manera general las principales características del ensayo y el procedimiento de inspección a desarrollar en el laboratorio del Comando Aéreo de Mantenimiento (CAMAN) de la Fuerza Aérea Colombiana. El método que se empleó en la investigación y en el desarrollo del proyecto fue de tipo documental, con el fin de establecer las normas y reglamentaciones requeridas para la certificación de la técnica en partículas magnéticas del laboratorio de pruebas no destructivas por la autoridad Aeronáutica. Finalmente, con el análisis de los métodos se elaboró el Manual de Procedimientos de Inspección con el propósito de certificar la técnica de partículas magnéticas.

Palabras clave: laboratorio de inspección NDT; pruebas NDT; técnica de partículas magnéticas.

Resumo: este artigo apresenta o procedimento geral para a utilização da técnica de partículas magnéticas; descreve em termos gerais as principais características do teste e do procedimento de inspeção a ser desenvolvidas no laboratório de CAMAN da Força Aérea Colombiana. O método utilizado na pesquisa e no desenvolvimento do projeto foi documental, a fim de estabelecer as regras e regulamentos necessários para a certificação da técnica em partículas magnéticas do laboratório de testes não destrutivas pela autoridade aeronáutica. Finalmente, com a análise dos métodos elaborou-se um Manual de Procedimentos de Inspeção com a finalidade de certificar a técnica de partículas magnéticas.

Palavras-chave: inspeção de laboratório NDT; testes END; técnica de partículas magnéticas.

Abstract: This article presents the general procedure for the use of the technique of magnetic particles; generally describes the main characteristics of the test and inspection procedure from CAMAN Lab developed at the Postgraduate School of the Colombian Air Force. The method used in the research and development of the project was documentary, in order to establish the rules and regulations required for certification of the magnetic particles technique in nondestructive testing laboratory by the Aeronautical Authority. Finally, based on the data analysis, an Inspection Procedure Manual was developed in order to certify the magnetic particles technique.

Key Words: Magnetic Particle Technique; NDT Inspection Lab; NDT tests. 


\section{Introducción}

El presente artículo tiene como objetivo presentar un procedimiento para evaluar la integridad de los componentes de las aeronaves con la técnica de inspección por Partículas Magnéticas vía húmeda, empleada para detectar discontinuidades superficiales. Asimismo, para satisfacer los requisitos y características de calidad que deben cumplir los materiales, equipos y herramientas utilizadas para la debida inspección, interpretación y evaluación, de acuerdo con los parámetros establecidos en los Manuales de Inspección aplicable de las Aeronaves y a la normativa internacional.

Por otro lado, busca evidenciar las variables que afectan la eficacia del ensayo y sus mecanismos de control. Debido a que los diferentes ensayos no destructivos tienen limitaciones de aplicación, profundidad, interpretación y sensibilidad para revelar las discontinuidades y defectos, se requiere determinar cuáles son los procesos de control de calidad aplicables que buscan minimizar las falencias de la aplicación de la prueba y así incorporarlas dentro del procedimiento descrito en este artículo y adicionarlo como a las tareas del programa de inspección de aeronaves.

De manera complementaria, se exploran las tendencias y los avances del ensayo de partículas magnéticas, como lo es el Procedimiento de Flujo Magnético (MFL) (Yanhua et al., 2011). Sin embargo, en el campo aeronáutico la aplicación del ensayo está limitada a lo establecido en los manuales de inspecciones específicos de las aeronaves y los nuevos procedimientos o variaciones al método que aún no han sido incorporados en los programas de mantenimiento.

\section{Método}

La descripción e interpretación de las características específicas de la técnica de partículas magnéticas, para su incorporación en un procedimiento de laboratorio, constituyó un proceso sistemático de investigación.

En concordancia se realizó un muestreo por conveniencia, el cual se define como un procedimiento en el que los investigadores teniendo en cuenta lo mencionado por Creswell (2008) y Llanos Pozzi (1994) seleccionaron el laboratorio de Inspecciones No Destructivas de CAMAN, por estar certificado ante la Unidad Administrativa Especial de la Aeronáutica Civil U.A.E.A.C., para realizar trabajos en las técnicas de inspección visual, ultrasonido y corrientes de Eddy bajo la norma ASTME 1316 (2012). Por otro lado, la detección y recolección de la información desarrollada dentro de esta investigación, constituye una parte importante porque la revisión y estudio documental de las técnicas NDT hicieron especial énfasis en la técnica de partículas magnéticas. Este proceso de detección y recolección de in- formación tuvo en cuenta la comparación y confrontación documental de cada norma, en las cuales no se va a profundizar; pero sí se concluye resaltando que se debe dar la debida comprensión de las necesidades de las normas ASTM E-1444-12 ASTM E-709-08, ASNT CP 189, ASNT SNT TC-1A y NAS 410.

Adicionalmente, las fuentes secundarias relacionadas tienen en cuenta los artículos indexados que referencian la importancia de emplear la técnica de partículas magnéticas.

Finalmente, para la consolidación de la información y aplicación de la técnica de partículas se analizaron los requerimientos propuestos por cada una de las normas seleccionadas y reglamentaciones requeridas para la certificación de la técnica en partículas magnéticas del laboratorio de pruebas no destructivas. De igual manera, se evaluó la capacidad instalada del laboratorio en cuanto a personal, instalaciones, herramientas, bancos, manuales, publicaciones técnicas y materiales.

\section{Apuntes a los métodos MFL vs PMFP}

Para abordar este tema primeramente se menciona a Infanzón y Romero (2003) quienes estudiaron la posibilidad de emplear un ensayo de partículas magnéticas de forma complementaria a ensayos usuales mecánicos y de reparación, para la detección de daños y efectos producidos en aceros al carbono en contacto con hidrógeno a altas temperaturas. A partir de este estudio, se reportó que mediante el ensayo de partículas magnéticas se pueden detectar diferentes tipos de fisuras y de magnitudes desiguales. Al mismo tiempo, detectaron mediante un ensayo radiográfico fisuras causadas en procesos de soldadura, defectos que se corroboraron mediante el ensayo de partículas magnéticas.

El método de partículas magnéticas asocia en su procedimiento el método de fuga de flujo magnético (MFL). Sin embargo, Yanhua et. al. (2011) señalan que este método presenta inconvenientes al igual que el método de corrientes de Eddy al ser aplicados en componentes como estructuras primarias de aeronaves, como son interacción complicada y perturbadora asociada a cambios y defectos en la geometría de la superficie, al tiempo que presenta la incapacidad para detectar defectos mutuamente orientados. Por esta razón, proponen el uso del método de perturbación permanente del campo magnético, mejor conocido como PMFP, el cual se muestra en la Figura 1. Este método permite determinar la presencia o ausencia de las discontinuidades en el material por medio de los cambios en la condición de contorno magnético que se producen debido a las discontinuidades geométricas en el material 


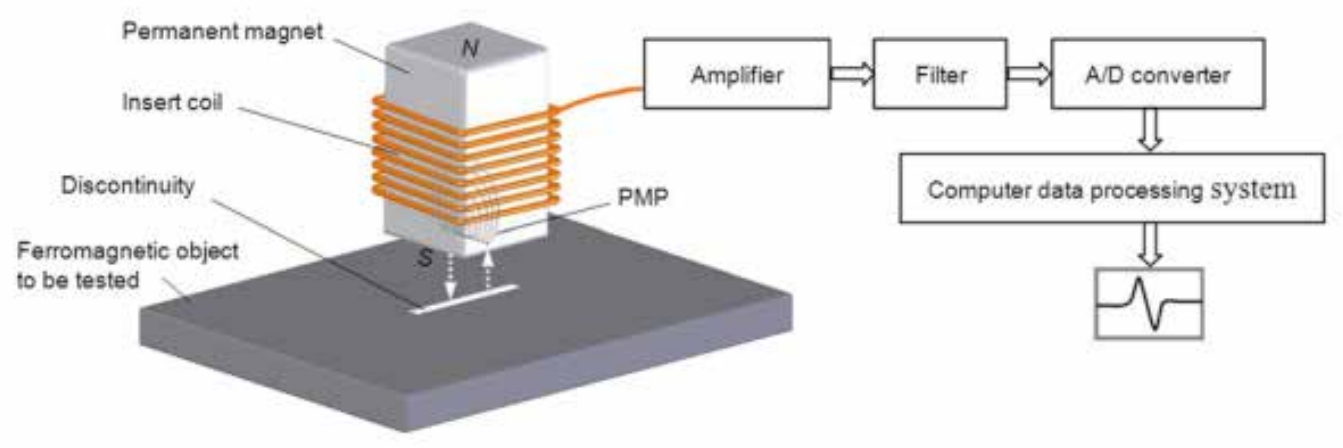

Figura 1. Esquema del sistema de perturbación permanente del campo magnético.

Fuente: Yanhua, Yihua, y Chen, (2011, p. 2), citado en Rincón, S. y Calvo, D. (2013, p.22). Manual de procesos de inspección de la técnica partículas magnéticas del laboratorio de CAMAN. Trabajo de Grado para optar por el título de Especialista en Logística Aeronáutica. Bogotá, Colombia: Instituto Militar Aeronáutico, ahora Escuela de Postgrados de la Fuerza Aérea Colombiana.

ferromagnético. Lo que mejora la aplicación en partes como soportes instalados en la raíz de plano, alojamiento de trenes y patín de cola de aeronaves.

En relación al tema, es necesario aclarar para el caso puntal de este artículo, el laboratorio de inspección NDT, el cual está ubicado en el Comando Aéreo de Mantenimiento (CAMAN), es la Unidad de mantenimiento a nivel mayor de la Fuerza Aérea Colombiana (FAC), donde a la fecha se emplea la aplicación de dos técnicas básicas del ensayo por partículas magnéticas como los son: el yugo magnético y el banco estático. En suma, las tendencias actuales indican que lo requerido en el campo de la ingeniería es incorporar la técnica de Perturbación Permanente del Campo Magnético- PMFP.

Debido a lo anterior, se hace necesario tener en cuenta lo siguiente: mediante el método de Fuga de Flujo Magnético-MFL no es posible comúnmente la detección de defectos paralelos a la dirección del campo magnético debido a la magnetización ortogonal. Es decir, la magnetización debe ser perpendicular a la orientación de defectos, lo que manifiesta una importante limitación para determinados usos que requiera este método de ensayo. Por tanto, el método de prueba MFL por exploración helicoidal no permite una alta velocidad de inspección y es limitada al aplicarla directamente en la aeronave. Sin embargo, el método PMFP tiene la capacidad de detectar defectos mutuamente orientados, dando lugar a una solución para los inconvenientes existentes en los métodos mencionados anteriormente. En resumen, la principal característica de PMFP es que depende de la perturbación magnética espacial causada por los defectos, resultando en una alta sensibilidad del método PMFP (Yanhua et al., 2011).

\section{Descripción de la inspección por partículas magnéticas}

El ensayo de partículas magnéticas de tipo No Destructivo, consiste en someter una pieza a una magnetización adecuada. Es uno de los ensayos más antiguos conocidos y en la actualidad se encuentra una gran variedad de aplicaciones en diferentes industrias. En la aviación es empleada por sus ventajas como son: metodología sencilla, fácil interpretación, economía y portabilidad, permitiendo el empleo de la técnica en componentes como por ejemplo: trenes de aterrizaje, bancadas de motor, fitting de sujeción de las estructuras primarias y pernos empleados en diferentes sistemas de la estructura. Se hace necesario tener en consideración que el principio físico en el que se basa la inspección por partículas magnéticas es el «magnetismo», fenómeno altamente abordado y del cual el artículo no tiene a objeto profundizar.

Continuando con lo expuesto y acorde a Obtesol (2007), Isotec (2010) y UNLP (2012) los componentes metálicos aeronáuticos ferromagnéticos y paramagnéticos, que presenten algún imperfecto en su superficie (de hasta 6 $\mathrm{mm}$ ), ya sean fisuras, poros, inclusiones no magnéticas en el material metálico, son susceptibles de ser inspeccionados por la técnica de partículas magnéticas.

Con este ensayo se pueden detectar discontinuidades generadas en la producción de piezas aeronáuticas y en fisuras generadas durante el servicio, como consecuencia a sobrecargas y ciclos de cargas como fatiga (Llog, 2010; UNLP, 2012) propias de las operaciones de aeronaves o componentes mayores como motores, hélices, trenes de aterrizaje, chapas de sujeción, rines, soportes de motor, pernos de sujeción de planos y superficies de control. 
Antes de continuar con la descripción de partículas magnéticas, se hace necesario presentar un breve marco de referencia, que aborde teóricamente las propiedades magnéticas con una descripción o acercamiento de los materiales. A continuación se encuentra una explicación de la inducción magnética y los materiales ferromagnéticos para una mejor comprensión del procedimiento aquí propuesto.

\section{Características de la inducción magnética}

La inducción magnética o densidad de flujo (B), es el número de líneas de fuerza, por unidad de área transversal a la dirección del flujo, inducidas por un campo magnético (H). La inducción "B" es proporcional a la intensidad de campo "H". Y el factor de proporcionalidad, denominado permeabilidad " $\mu$ ", representa la mayor o menor dificultad con que un material puede ser magnetizado. Cuanto más magnético sea un material más "permeable" será al flujo magnético y por lo tanto permitirá pasar una mayor cantidad de líneas de fuerza por unidad de área (CETP/UTU, 2011, p. 28).

Matemáticamente esta relación entre campo magnético e inducción se expresa en la Ecuación 1:

$$
B=\mu^{*} H
$$

Las propiedades magnéticas de las sustancias tienen su origen en el movimiento de los electrones y en los momentos magnéticos permanentes de los núcleos y de los electrones. Un modelo elemental de la estructura del átomo considera que los electrones viajan en órbitas en torno del núcleo (niveles de energía). En este modelo, el movimiento de un electrón constituye una espiral de corriente, y por lo tanto genera un campo magnético. Además del momento magnético orbital, el electrón posee un momento magnético intrínseco. Este último, se refiere a menudo como el momento magnético de espín del electrón, ya que una forma de imaginar su origen es considerar al electrón como una carga que "gira" en torno a su eje. Como tal, el electrón actuaría en una espiral de corrientes. Excepto para los elementos de transición, los momentos magnéticos del espín de los electrones de los átomos se cancelan por pares hasta donde es posible. Es decir, los momentos de espín del electrón se orientan en direcciones opuestas para los dos electrones de un par (CETP/UTU, 2011).

Para Castaño (2012 p.1) Los tipos de ordenaciones de spins electrónicos que dan lugar a un momento magnético espontáneo (excepto en el caso del anti-ferromagnético simple) se muestran en la Figura 2.

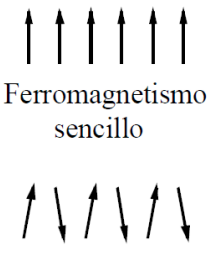

Antiferromagnetismo

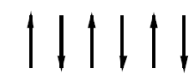

Antiferromagnetismo sencillo

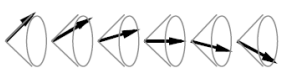

Disposición helicoidal de spin

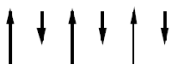

Ferrimagnetismo

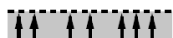

Banda de energía ferromagnética
Figura 2. Ordenación de spins electrónicos.

Fuente: Castaño Carmona, (2012, p. 1), citado en Rincón, S. y Calvo, D. (2013). Manual de procesos de inspección de la técnica partículas magnéticas del laboratorio de CAMAN (p.22). Trabajo de Grado para optar por el título de Especialista en Logística Aeronáutica. Bogotá, Colombia: Instituto Militar Aeronáutico, ahora Escuela de Postgrados de la Fuerza Aérea Colombiana.

Entonces, los materiales magnéticos pueden clasificarse en función de su permeabilidad; los ferromagnéticos son todos aquellos materiales cuya permeabilidad sea mayor a 1, los paramagnéticos son todos aquellos en los cuales su permeabilidad presenta un valor semejante al del aire, y aquellos materiales cuya permeabilidad es menor que la del aire se denominan Diamagnéticos. Esto se resume a continuación de forma numérica a partir de lo encontrado en CETP/UTU (2011, p. 29):

\section{Ferromagnéticos $\mu>>1$ \\ Paramagnéticos $\mu \approx 1$ \\ Diamagnéticos $\mu<1$}

Según Castaño (2012) empleando un imán, se puede lograr diferenciar fácilmente entre cada tipo de material, ya que los materiales ferromagnéticos son fuertemente atraídos. El momento magnético se asocia con una carga moviéndose en una trayectoria cerrada, y se debe principalmente a 3 efectos:

- El cambio de momento angular orbital producido al aplicar un campo magnético

- El momento angular orbital de los electrones alrededor del núcleo

- El spin de los electrones

\section{Materiales paramagnéticos}

Se hace necesario retomar lo planteado por Castaño (2012) y complementado en lo encontrado en CETP/ UTU (2011 p. 31-32), quienes informan que en un átomo, los únicos electrones que pueden contribuir al momento magnético total son los que están en capas incompletas, generalmente son los electrones de valencia, dado que en las capas electrónicas completas el momento magnético orbital y de spin es cero. Como la mayoría de los átomos tienen capas incompletas, también tendrán 
CIENCIA Y PODER AÉREO | Revista Científica de la Escuela de Postgrados de la Fuerza Aérea Colombiana | Vol. 10 | Enero - Diciembre de 2015

un momento magnético no nulo. Pero esto sólo es posible en átomos libres y no para átomos situados en una red cristalina, los cuales se encuentran ligados entre sí por fuerzas de enlace. La razón es que la energía de canje de los electrones de átomos vecinos es normalmente mínima cuando sus spines están dispuestos de forma anti-paralela y de ahí que el momento dipolar total de la molécula sea nulo.

Además, en los metales se encuentra el comportamiento paramagnético debido a los electrones de conducción. Este tipo de paramagnetismo muestra la propiedad de que la susceptibilidad es prácticamente independiente de la temperatura. Los materiales empleados para aplicaciones prácticas están hechos de sales de hierro o de tierras raras (Castaño Carmona, 2012, p. 5).

\section{Materiales ferromagnéticos}

Castaño $(2012$, p. 2) indica como características particulares de los materiales ferromagnéticos, que:

- En los materiales ferromagnéticos se observa una imanación espontanea " $M_{s}$ " en ausencia de un campo magnético externo.

- La imanación espontanea varía con la temperatura alcanzando un máximo para $\mathrm{T}=0{ }^{\circ} \mathrm{K}$ y presentando una disminución continua con el incremento en la temperatura, cayendo hasta 0 para una temperatura "TC" denominada temperatura de Curie ferromagnética.

- Para T>Tc el material experimenta una transición de fase ferromagnética-paramagnética con una susceptibilidad en la fase paramagnética dada por la relación:

$$
\mathrm{X}=\mathrm{C} /\left(\mathrm{T}-\mathrm{T}_{-} \mathrm{C}\right)
$$

- Donde $X$ no está definida para $T<T c$ donde el material presenta imanación espontanea.

- Un material ferromagnético no tiene una imanación espontanea en la misma dirección a lo largo de todo su volumen, sino que presenta regiones más pequeñas de imanación de dirección uniforme llamadas dominios magnéticos y están orientadas al azar.

- Los únicos elementos ferromagnéticos son: hierro (Fe), cobalto (Co), niquel (Ni), gadolinio (Gd) y disprosio (Dy).

En materiales ferromagnéticos y para $T<<T_{c^{\prime}}$ los momentos magnéticos de los átomos, a escala microscópi- ca, están todos alineados. Sin embargo, a una escala más amplia, el momento magnético de la muestra puede ser inferior e incluso nulo. Por lo cual, se hace necesario, para alcanzar la imanación de la muestra, aplicar un campo magnético exterior. Esto tipo de pruebas según Castaño (2012) resulta eficaz tanto para mono-cristales como para muestras poli-cristalinas.

Asimismo, Castaño y Carmona (2012) explican que dicho "fenómeno se basa en que los materiales ferromagnéticos macroscópicos están compuestos de un gran número de pequeñas regiones o dominios magnéticos en cuyo interior la imanación local está saturada" (p. 1) Para una mejor comprensión del tema ver Figura 3.

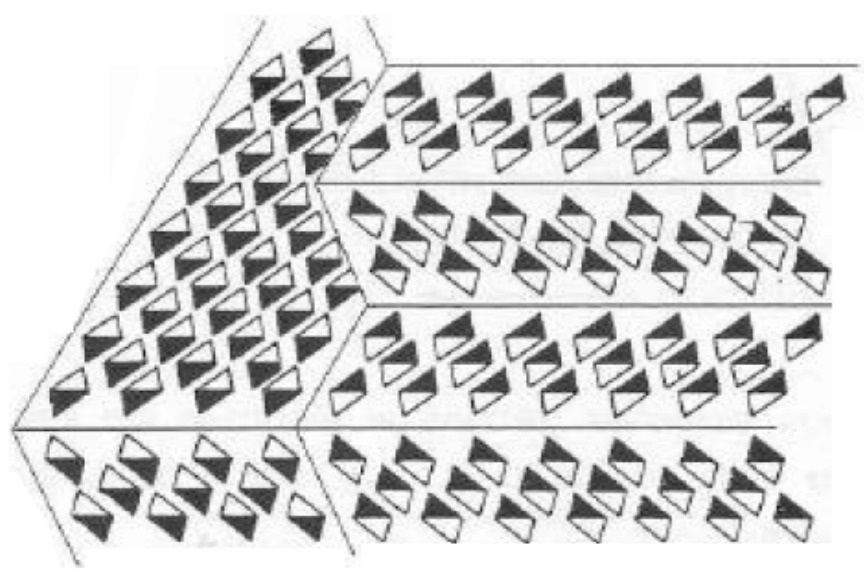

Figura 3. Dominios magnéticos con imanación de saturación en diferente dirección es un material ferromagnético.

Fuente: Castaño (2012, p. 1), citado en Rincón, S., y Calvo, D. (2013). Manual de Procesos de Inspección de la Técnica Partículas Magnéticas del Laboratorio de CAMAN (p. 22). Trabajo de Grado para Optar por el título de Especialista en Logística Aeronáutica. Bogotá, Colombia: Escuela de Postgrados de la Fuerza Aérea

En concordancia, al colocar ésta en un campo magnético, la imantación resultante puede aumentar de dos formas distintas, por crecimiento de dominios favorablemente orientados respecto al campo externo (ver Figura 4 (a)), y por rotación de la dirección de imantación hacia la dirección del campo externo (ver Figura 4 (b)).

Finalmente, resulta adecuado considerar lo mencionado por Castaño y Carmona (2012) en complemento con lo expuesto por la CETP/UTU (2011) donde en la Figura 4 (a) se observa que en los campos externos débiles el volumen de los dominios está orientado favorablemente con respecto al campo externo, mientras que en la Figura 3 (b) los campos externos intensos la imanación gira en la dirección del campo externo. 
a)

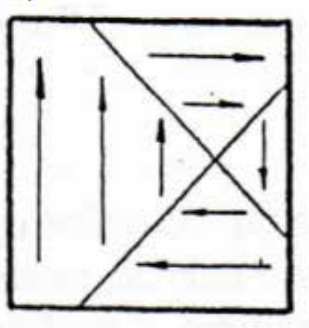

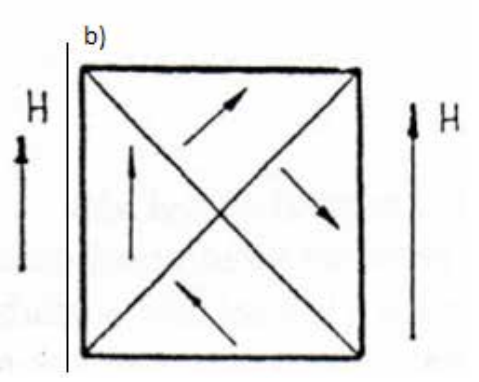

Figura 4. (a), Imantación por crecimiento de dominios; (b), Imantación por rotación.

Fuente: CETP/UTU (2011, p. 33), citado por Rincón, S. y Calvo, D. (2013) Manual de Procesos de Inspección de la Técnica Partículas Magnéticas del Laboratorio de CAMAN (p. 22). Trabajo de Grado para Optar por el título de Especialista en Logística Aeronáutica. Bogotá, Colombia: Escuela de Postgrados de la Fuerza Aérea.

\section{Ensayo por partículas magnéticas en laboratorio}

Como es conocido, el ensayo por partículas magnéticas consta de dos métodos de ensayos no destructivos planteado por Muñoz (2002) y AEND (2002) respectivamente, el primero es ensayo de fuga de flujo magnético y el segundo es el ensayo visual el cual como su nombre lo indica consiste en identificar de manera visual las proporciones de fuga magnética dentro del mismo.

Por tanto, en esta sección del artículo se presenta un procedimiento para la adición de partículas magnéticas al laboratorio del CAMAN, el cual comprende la inspección mediante el empleo de la técnica no destructiva de Partículas Magnéticas vía húmeda (MT), cuyo alcance va hasta detectar discontinuidades superficiales y subsuperficiales, que pueden afectar la integridad de materiales ferromagnéticos, esta inspección puede aplicarse como control de calidad a los procesos de fabricación y/o para detectar grietas en componentes aeronáuticos de aeronaves de la FAC y/o la aviación comercial. Por tanto, es necesario cumplir con las recomendaciones de inspección dadas en los documentos de los fabricantes de las aeronaves y/o dentro de lo mencionado de sus componentes aeronáuticos. Dada la importancia de este procedimiento para el laboratorio, es necesario mencionar que para el establecimiento de la adición de la técnica de partículas magnéticas se tuvo en cuenta la normatividad relacionada en la Figura 5.

\section{Equipos y materiales del ensayo de partículas magnéticas}

Es indispensable que al momento de realizar el ensayo, los equipos se encuentren verificados, calibrados y certificados según sean sus requerimientos. Asimismo, se debe contar con yugos magnéticos y un banco horizontal estático de corriente eléctrica, que a continuación se describen:

- ASTM E-1444-12 última revision. Standard Practice for Magnetic Particle Examination.

- $\quad$ ASTM E-709-08 última revision. Guide for Magnetic Particle Examinations.

- $\quad$ Norma ASTM E 1316-13a. Terminology for Nondestructive Examinations.

- $\quad$ Recommended Practice for Nondestructive Testing Personnel Qualification and Certification. Última revision. ASNT SNT-TC-1A.

- $\quad$ AIA Standard NAS 410 NAS Certification and Qualification of Nondestructive

- $\quad$ Testing Personnel.

- Práctica Escrita de Entrenamiento, Calificación y Certificación para el Personal de Ensayos No Destructivos (NDT) del Taller de inspección y análisis del CAMAN Servicios Especializados Clase I.

- T.O. 33B-1-1 Nondestructive Methods, Basic Theory

- Manuales de Ensayos No Destructivos de los fabricantes aplicables a aeronaves de la Fuerza Aérea Colombiana y de la Aviación Comercial.

Figura 5. Documentos de Referencia para el establecimiento del Ensayo de Partículas Magnéticas.

Fuente: Tomado y adaptado de Rincón y Calvo (2013). Manual de Procesos de Inspección de la Técnica Partículas Magnéticas del Laboratorio de CAMAN (pp. 81-82). Trabajo de Grado para Optar por el título de Especialista en Logística Aeronáutica. Bogotá, Colombia: Escuela de Postgrados de la Fuerza Aérea

\section{Equipos}

\section{Yugo electromagnético}

Se deben utilizar yugos electromagnéticos de corriente alterna o directa, los yugos magnéticos permanentes de corriente $\mathrm{AC}$ son requeridos para levantar un peso de 4.5 Kg con una distancia entre polos de $300 \mathrm{~mm}$ o menor y una fuerza de separación del yugo de $2.25 \mathrm{Kg}$ y los yugos de DC o imanes permanentes se requiere si la distancia entre los polos es menor a $75 \mathrm{~mm}$. El campo magnético generado por estos yugos, son inducidos por corriente continua y corriente alterna (AC/DC).

Banco partículas magnéticas (magnetización mediante corriente eléctrica)

Equipo estacionario de marca ELECTROFLUS Modelo TMX2020- con capacidad en DC 6000 y AC 3.000 AMPE- 
CIENCIA Y PODER AÉREO | Revista Científica de la Escuela de Postgrados de la Fuerza Aérea Colombiana | Vol. 10 | Enero - Diciembre de 2015

RIOS. El equipo debe estar provisto de un amperímetro debidamente calibrado, un control selector de corriente y un control de corte rápido no menor a medio segundo. Este equipo cumplirá con los requerimientos de calibración establecidos en ASTM E1444.

\section{Unidad horizontal del banco estático de partículas magnéticas}

Unidad horizontal apropiada para utilizar partículas húmedas fluorescentes suspendidas en Carrier II. Esta unidad estará instalada en un cuarto oscuro. De igual forma, tendrá un sistema de recirculación continua, que permita mantener la adecuada agitación de las partículas magnéticas y en forma óptima la aplicación del baño a través de rociado.

\section{Materiales}

Los materiales requeridos para la realización de la inspección por partículas magnéticas son las partículas húmedas fluorescentes, empleadas para detectar defectos superficiales, en especial fisuras causadas por los ciclos de carga a los que es sometido material durante su vida en servicio, o como consecuencia de las fallas en el proceso de fabricación del componente.

Partículas magnéticas: empleadas para estas inspecciones son las de naturaleza húmeda y fluorescentes, que deberán cumplir con las normas y requerimientos de las normas AMS 3041, ANS 3042, AMS 3043, AMS 3044, AMS 3045 o AMS3046; deben tener alta permeabilidad para permitir facilidad de magnetización y atracción hacia la discontinuidad, y baja retentividad de modo que no sean atraídas entre sí para evitar aglomeración magnética. Las partículas no deben ser tóxicas, deben estar libres de óxido, grasa, pintura, suciedad y otros materiales extraños que puedan interferir su uso. Estas deben ser utilizadas de acuerdo con las instrucciones del fabricante. Se deberá disponer de partículas vía húmeda en aerosol y partículas magnéticas Magnaglo 14 A del tipo fluorescente húmedo, suspendidas en Magnaglo Carrier II. De igual forma, la concentración será controlada dentro de los parámetros establecidos por (ASTM E1444 / E1444M, 2012), para partículas húmedas fluorescentes; esta concentración, estará dentro del rango de 0.1 a $0.4 \mathrm{ml}$ en $100 \mathrm{ml}$ de muestra tomada del baño de partículas fluorescentes a utilizar. Su aplicación será por rociado sobre la superficie de la pieza.

Indicadores de campo magnético: se utilizará un indicador de intensidad de campo tipo torta (MagnetotestPenetrameter or Magnetic Field Indicator) con defectos artificiales, que permitan evidenciar la presencia y la dirección aproximada del campo magnético. A continuación en la Tabla 1 se describen los indicadores propuestos para la adición de partículas magnéticas.
Tabla 1. Indicadores de Campo Magnético para la Adición de Partículas Magnéticas

\begin{tabular}{|c|c|}
\hline \multicolumn{2}{|r|}{ Indicadores } \\
\hline $\begin{array}{l}\text { Pie Magnetic } \\
\text { Fiel Indicator }\end{array}$ & $\begin{array}{l}\text { Indica la dirección del flujo del } \\
\text { campo magnético externo. }\end{array}$ \\
\hline $\begin{array}{l}\text { Campo Magnético } \\
\text { Residual }\end{array}$ & $\begin{array}{l}\text { Indica la fuerza residual del campo } \\
\text { magnético de la pieza a inspec- } \\
\text { cionar de } \pm 1.5 \text { gauss por división } \\
\text { o menor, con un rango total de } \\
\text { escala de }-10 \text { a }+10 \text { gauss }\end{array}$ \\
\hline Efecto Hall & $\begin{array}{l}\text { Mide la fuerza del campo mag- } \\
\text { nético que se genere en mag- } \\
\text { netización circular (conductor } \\
\text { por cabezotes) y magnetización } \\
\text { longitudinal (bobina). Valores en } \\
\text { un rango de } 30 \text { a } 60 \text { Gauss (2,4 a } \\
\text { 4,8KA/m). }\end{array}$ \\
\hline $\begin{array}{l}\text { Calidad Cuantitativa y } \\
\text { Bloque de Patrón (QQI) }\end{array}$ & $\begin{array}{l}\text { Indica discontinuidades y la sen- } \\
\text { sibilidad de las partículas magné- } \\
\text { ticas. }\end{array}$ \\
\hline
\end{tabular}

Fuente: Tomado y adaptado de Calvo y Rincón (2013). Manual de Procesos de Inspección de la Técnica Partículas Magnéticas del Laboratorio de CAMAN (pp. 81-82). Trabajo de Grado para Optar por el título de Especialista en Logística Aeronáutica. Bogotá, CoIombia: Instituto Militar Aeronáutico, Fuerza Aérea Colombiana.

Tubo centrífugo: empleado para determinar el nivel de concentración de las partículas húmedas fluorescentes, se debe contar con un tubo centrífugo tipo pera de $100 \mathrm{ml}$, cuyo vástago es de $1.0 \mathrm{ml}$ con divisiones de $0.05 \mathrm{ml}$.

Fuente de luz negra (luz ultravioleta): fuente de luz ultravioleta o lámpara de luz negra tendrá una intensidad sobre el área a inspeccionar de $1000 \mu \mathrm{W} / \mathrm{cm}^{2}$ a 15 pulgadas de distancia.

Medidores de luz negra: un medidor de luz negra garantiza que la intensidad de la luz negra utilizada no sea menor a la mínima requerida para la inspección por partículas fluorescentes (1000 $\mu \mathrm{W} / \mathrm{cm} 2$ a 15 Pulgadas). Este instrumento deberá estar calibrado debidamente.

Desmagnetizador: el equipo de desmagnetización consiste de una bobina de 11 pulgadas de diámetro de corriente AC donde la pieza es desmagnetizada retirándola lentamente de adentro hacia fuera de la bobina y comprobando su campo residual con un indicador de campo. 
Procedimiento adición de la técnica de partículas magnéticas basado en Llog (2010) Obtesol (2007) y Isotec (2010)

Antes de desarrollar un ensayo por la técnica de partículas magnéticas es necesario verificar que el área o la zona donde se va a efectuar la inspección y evaluación estarán debidamente oscurecidas, de manera que reduzca el fondo de luz visible hasta el nivel más bajo posible (no mayor a 20 Lx o 2 fc). Esta zona dispondrá de una lámpara de luz negra que garantice una intensidad mínima de 1000 $\mu \mathrm{W} / \mathrm{cm} 2$ en la superficie de la pieza bajo examen, cuando esté a una distancia de 15 pulgadas.

La superficie a examinar, estará seca y libre de grasa, pintura, óxidos, o de cualquier otra sustancia extraña o suciedad que pueda dar lugar a falsas indicaciones o resultados poco seguros. El acabado superficial de las piezas será tal, que permita la interpretación adecuada de las indicaciones propias del ensayo. Por lo tanto, la superficie del material a inspeccionar no presentará irregularidades superficiales agudas y estará libre de mordeduras o cambios bruscos superficiales.

Paso 1) Pre-inspección de la superficie. Verificar con el gaussometro la presencia de campo residual, producto de una magnetización previa, que pueda interferir con el ensayo. Si la pieza o parte retiene un campo magnético residual significativo (mayor a 3 gauss) se debe desmagnetizar antes de continuar con la nueva magnetización.

Paso 2) Magnetización. Esta primera etapa tiene por finalidad crear en la pieza, o introducir en ésta un campo magnético de intensidad y dirección conocidas. Estos campos magnéticos se pueden crear mediante el yugo magnético o el equipo estático de partículas magnéticas. En el examen de un objeto por partículas magnéticas, en el caso que se trabaje con banco de partículas (corriente eléctrica), la aplicación de las partículas podrá ser realizada mientras está circulando la corriente de magnetización o después que esta haya cesado.

Paso 3) Aplicación de las partículas magnética. Después de la magnetización de la pieza, se debe proceder a la aplicación de las partículas magnéticas sobre la superficie de interés con el propósito de identificar los campos de fuga que se originan a causa de la presencia de discontinuidades. Este procedimiento puede realizarse manualmente o por métodos mecánicos, siendo éste último usado en la mayoría de inspecciones en las que se emplee un banco de partículas y partículas magnéticas por vía húmeda.
Paso 4) Interpretación. Las interpretaciones de la inspección sólo podrán ser realizadas por personal técnico NDT Calificado y Certificado como Nivel II o III en Ensayos No Destructivos de acuerdo con la Práctica Escrita CAMANNDT-PECCP del Taller Aeronáutico de Reparaciones CAMAN. Es necesario recordar que se debe hacer una correcta interpretación de las indicaciones proporcionadas, por cualquiera que sea el método de ensayo no destructivo que se utilice, es aconsejable:

i. Conocer la naturaleza del material que constituye la pieza objeto del ensayo, así como el historial de fabricación de la misma (proceso de fabricación y tratamientos térmicos a que ha sido sometida).

ii. Tener en cuenta heterogeneidades o discontinuidades que con carácter específico pueden presentarse con más frecuencia en cada una de las etapas de fabricación.

iii. Efectuar, si se considera necesario, ensayos complementarios, aplicando cualquier otro método de ensayo con el fin de disponer de información suficiente para la identificación de la indicación.

Por cuanto se refiere al método de ensayo por partículas magnéticas, se puede afirmar que en la mayoría de los casos, las indicaciones que proporciona son nítidas y claramente definidas siempre que procedan de discontinuidades, tales como grietas superficiales, cuyo plano sea perpendicular a las líneas de fuerza del campo magnético. En cambio, si las discontinuidades son subsuperficiales, sus correspondientes indicaciones aparecerán con sus bordes más difusos.

Paso 5) Desmagnetización. Las piezas a inspeccionar de naturaleza ferromagnética, después de haber sido sometidas a un campo magnético y una vez que ha cesado su acción, presentan un cierto magnetismo residual o remanente, cuya intensidad dependerá de la retentividad del material. Este magnetismo remanente puede ser despreciable para materiales tales como aceros blandos de bajo contenido en carbono (baja retentividad), mientras que puede alcanzar valores altos en materiales duros, pudiendo llegar a ser del orden de magnitud de los valores que se pueden alcanzar en las aleaciones especiales.

En el campo aeronáutico se debe tener en cuenta que el magnetismo remanente puede afectar el correcto funcionamiento de los equipos o aparatos incorporados en la estructura donde va montada la pieza, parte o componente examinado.

Paso 6) Limpieza final. Una vez terminada la inspección de la pieza, se debe efectuar la remoción de las 
CIENCIA Y PODER AÉREO | Revista Científica de la Escuela de Postgrados de la Fuerza Aérea Colombiana | Vol. 10 | Enero - Diciembre de 2015

partículas acumuladas antes que se seque el medio líquido sobre la superficie se puede efectuar con solvente o aire.

En caso de ser requerido se deberán proteger las partes de cualquier posible corrosión o daño durante el proceso de limpieza y tratadas para prevenir la ocurrencia de corrosión después de la inspección final.

Paso 7) Reporte. Finalmente, es necesario realizar el Reporte de Ensayo No Destructivo donde se consigne la identificación completa del componente, Método de Inspección MT partículas magnéticas, Resultado del procedimiento NDT (número, clasificación y localización de discontinuidades), Nombre y firma del Inspector NDT con Nivel de Certificación y número de Autorización, así como se debe consignar los Criterios de aceptación y rechazo.

\section{Nota: Criterios de aceptación}

Se debe indicar solamente la condición encontrada, es decir, la descripción de la discontinuidad o defecto. De acuerdo con esto, la pieza o elemento puede ser aceptable o rechazable únicamente por inspección. Cualquier defecto encontrado, por pequeño que sea deberá ser rechazado por Inspección No Destructiva. Aunque también existen parámetros estándar dados por las normas ASTM en función del tipo de material y magnitudes máximas permisibles en proporción al tamaño del objeto que se pueden tomar como sugerencia, es importante recurrir en primera instancia a los manuales de inspección aplicable.

\section{Aplicaciones del ensayo}

Como es conocido, el método de inspección por partículas revela de un mejor modo la posición de discontinuidades que se encuentran perpendiculares a las líneas de campo, de tal forma que fisuras orientadas en dirección paralela a las líneas de campo no se visualizarán, por tal razón debe practicarse como mínimo dos magnetizaciones a la pieza a fin de encontrar todas las discontinuidades presentes; las magnetizaciones se realizarán de tal manera que la segunda magnetización dé lugar a líneas de campo perpendiculares a las de la primera en el caso de magnetización con yugos. En el caso de que el proceso de magnetización se realice en un banco de partículas posiblemente sea suficiente con una magnetización circular y una longitudinal. En ambos casos éstas se realizarán separadas la primera de la segunda por las etapas de aplicación de las partículas y su respectiva observación.

Este es el uso más amplio que presenta este ensayo, dado que quiere asegurar que el material desarrollado no presenta defectos que puedan interfieran en su funcionamiento (Obtesol, 2007, p. 1; Llog, 2010, p. 1). Es la categoría más continuamente empleada en el proceso de aeronave- gabilidad continuada de las aeronaves, ya que partes de los motores, conjuntos de trenes, componentes dinámicos de helicóptero, y estructuras metálicas son sometidas al ensayo para garantizar su integridad estructural.

\section{Conclusiones}

Al someter un material ferro-magnético a un campo magnético, los dominios se orientan o alinean de forma paralela al campo magnético externo, produciendo así un imán. Cuando se presenta este tipo de orientación inducida, el material desarrolla una fuerza total equivalente a la suma de todos los dominios, conocida como "Flujo Magnético" la dirección de este campo es definida por la dirección de las líneas de fuerza y como características principales presentan lo siguiente: a) una dirección definida, partiendo del polo Norte hasta el polo Sur y continúan así su camino a través del imán, b) una trayectoria continua formando siempre una curva o circuito cerrado de forma individual lo que quiere decir que no se cruzan ni se unen entre sí, y c) la densidad del campo disminuye al aumentar su distancia de los polos y siguen los caminos de menor resistencia magnética.

Por otra parte, la investigación arrojo la generación de un procedimiento de inspección para la técnica de partículas magnéticas, complementando los procesos existentes del Manual de Procedimientos de Inspección del TAR CAMAN ante la autoridad Aeronáutica.

El empleo de un procedimiento establecido y soportado en las normas internacionales y como complemento a los manuales de fabricantes y/o de mantenimiento de las aeronaves, asegura el cumplimiento de los procedimientos requeridos para garantizar la aeronavegabilidad continuada de los componentes aeronáuticos.

Finalmente, se sugiere que se continúe investigando y desarrollando adiciones a la técnica de partículas magnéticas empleadas por la FAC, de tal manera que sirva de apoyo en el desarrollo de la industria aeroespacial.

\section{Agradecimientos}

Agradecemos al Comando Aéreo de Mantenimiento - CAMAN y al Departamento de Investigación de la Escuela de Postgrados de la Fuerza Aérea Colombiana. También un especial agradecimiento al CT. Iván Fernando Rodríguez Pineda, al Ingeniero PhD cand. Cesar Augusto Rodríguez Adaime, Ingeniero Especialista Nivel II ASNT Asdrúbal Góngora y Mg. Mayden Yolima Solano Jiménez quienes con sus conocimientos contribuyeron a la investigación. La más sincera gratitud a cada una las personas que aportaron en 
su campo de desarrollo, para que este trabajo de investigación pudiera llegar a culminarse de modo satisfactorio.

\section{Referencias}

AEND. (2002). Particulas Magnéticas Nivel II (End: Ensayos No Destructivos) (Vol. 1). Madrid, España: FUND. CONFEMETAL.

Arango, A. (2011). Análisis de Datos Cualitativos. Medellín, Antioquia, Colombia.

ASNT CP-189. (2006). Standard For Qualification And Certification Of Nondestructive Testing Personnel. 2006. Norma, United states .

ASTM E 1316. (2012). Standard Terminology for Nondestructive Examinations. Normas técnicas, ASTM INTERNATIONAL, United States.

ASTM E 709. (2008). Standard Guide for Magnetic Particle Examinations. ASTM E 709. Norma Internacional, ASTM INTERNATIONAL, United States.

ASTM E1359. (2012). Norma, ASTM Internatonal.

ASTM E1444 / E1444M. (2012). ASTM E1444 / E1444M - 12 Standard Practice for Magnetic Particle Testing. Normas técnicas, ASTM Internatonal, EEUU.

ASTM E543. (2013). Standard Specification for Agencies Performing Nondestructive Testing. Norma, ASTM International, EEUU.

Berhsa. (2007). Artículo 7. [En línea] Recuperado el 5 de febrero de 2013, de scribd: http://es.scribd.com/doc/98388337/Articulo-7-Ensayo-Particulas-Magneticas

Castaño Carmona, E. (2012). Tecnun. Campos tecnológico Universidad de Navarra. Recuperado el 19 de febrero de 2013, de Tecnun. Campos tecnológico Universidad de Navarra: http:// www.tecnun.es/asignaturas/PFM_Mat/Prog/Dia_Para.pdf

Castaño Carmona, E. (2012). Tecnun. Campos tecnológico Universidad de Navarra. Recuperado el 19 de febrero de 2013, de Tecnun. Campos tecnológico Universidad de Navarra: http:// www.tecnun.es/asignaturas/PFM_Mat/Prog/Ferrom.pdf

Castaño Carmona, E. (2012). Tecnun. Campus tecnológico Universidad de Navarra. Recuperado el 19 de febrero de 2013, de Tecnun. Campus tecnológico Universidad de Navarra: http:// www.tecnun.es/asignaturas/PFM_Mat/Prog/Matmagv2.pdf

CETP/UTU. (2011). Partículas Magnetizables. CETP/UTU, Ingeniería. Montevideo Urugay: Servicio de Mateniemiento.

Creswell, J. W. (2008). Muestreo por conveniencia.

De Debold B., V. D., y William J., M. (1971). Manual de técnica de la investigación educacional.

Echeverria, R. (2002). Partículas Magnetizables. Laboratorio NDT, Universidad Nacional de Comahue.

Equipos y Laboratorios de Colombia, S. (2011). Equipos y Laboratorios de Colombia. Recuperado el 14 de febrero de 2013, de
Equipos y Laboratorios de Colombia: http://www.equiposylaboratorio.com/sitio/contenidos_mo.php?it=5079

Fuerza Aérea Colombiana (2008). Manual de Mantenimiento. Bogotá Colombia.

FuerzaAérea Colombiana. (2011). Partículas Magnetizables. Servicio de Mateniemiento - Dpto de Ingeniería. Bogotá Colombia

Gonzales, J. (7 de mayo de 2011). scribd. Recuperado el 13 de febrero de 2013, de scribd: http://es.scribd.com/doc/59384227/ PARTICULAS-MAGNETICAS-COMPLETO

Hernández Sampieri, R., Fernández-Collado, C., \& Baptista Lucio, P. (2007). Metodología de la investigación (Cuarta ed.). México, México: McGraw-Hill Interamericana.

Industrial Aeronautica. (2004). Partículas Magnéticas. Curso de Ensayos no destructivos.

Infanzón, S., y Romero, J. (2003). Los Ensayos No Destructivos en la detección de ataque por hidrógeno a alta temperatura. Conferencias PANNDT 2003, Rio de Janeiro.

Isotec. (2010). Inspección y Diagnóstico Técnico. Recuperado el 24 de enero de 2013, de Isotec: http://www.isotec.com.co/

Llanos Pozzi, M. J. (1994). Aproximaciones cualitativas y cuantitativas . En J. W. Creswell, Diseño de investigación. (pp. 143-171). Buenos Aires, Argentina: Sage.

Llog. (2010). Ilog S.A. Recuperado el 24 de enero de 2013, de http://www.llogsa.com/

Muñoz Abella, M. (2002). Mantenimiento Industrial. Informes de lectura de clase, Universidad Carlos III de Madrid, Área de Ingeniería Mecánica, Madrid.

Mantenimiento Industrial. (2002). Recuperado el 15 de febrero de 2013, de Scribd: http://es.scribd.com/doc/39863503/Particulas-magneticas

Obtesol. (27 de febrero de 2007). Observatorio Tecnológico de la Soldadura. Recuperado el 24 de enero de 2013, de Obtesol: http://www.obtesol.es/

RAC. (2011). Reglamento Aeronáutico Colombiano RAC. Unidad Administrativa Especial de la Aeronáutica Civil Colombiana. Colombia: Aeronáutica Civil.

UNLP. (2012). Partículas Magnéticas. Cátedra-Ensayos no Destructivos. (pp. 1-25). La plata: Departamento de Aeronáutica.

Yanhua, S., Yihua, K., \& Chen, Q. (2011). A new NDT method based on permanent magnetic field perturbation. NDT\&E International (44), 7. 


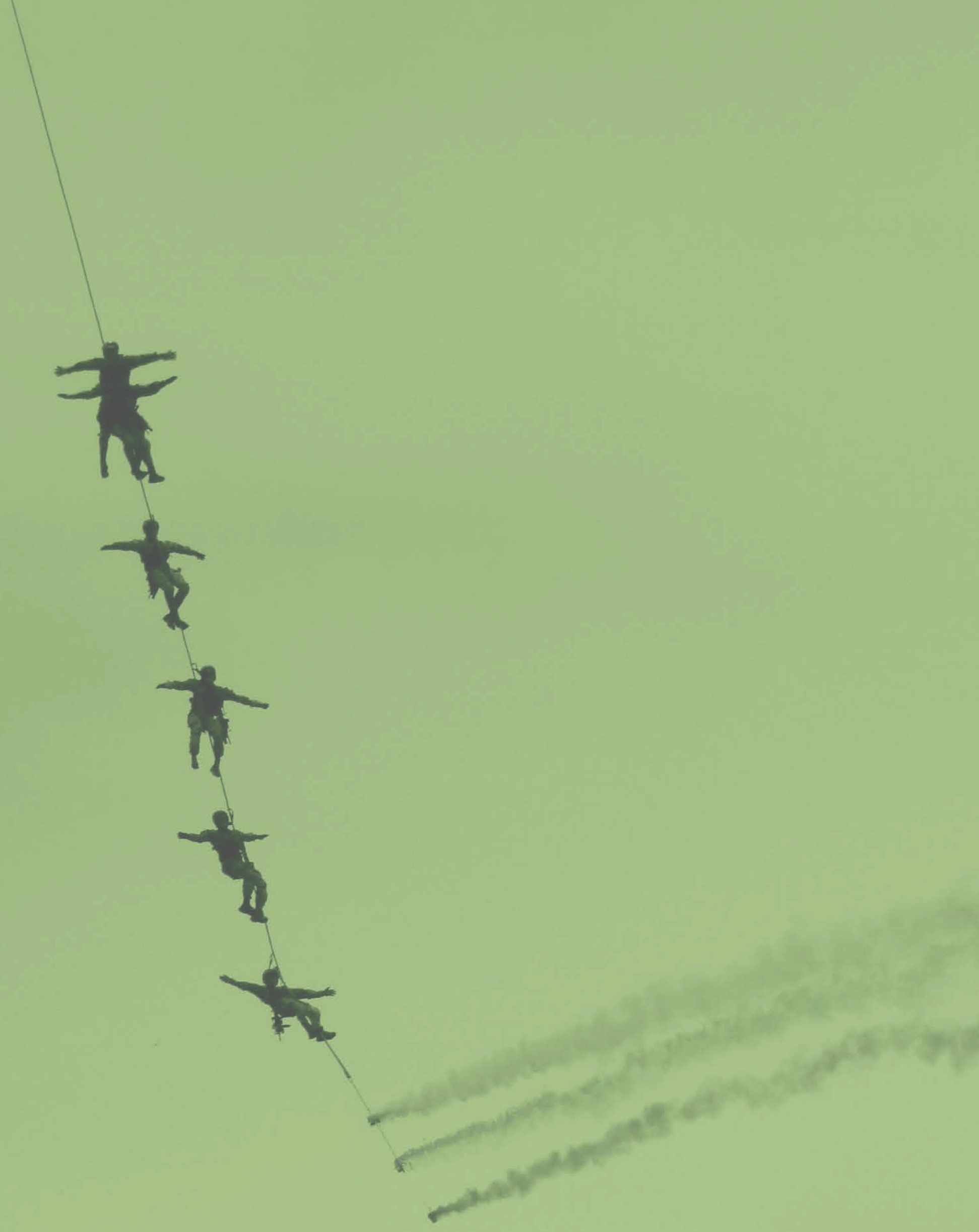

